\title{
Removing Redundancy in Partial Discharge Cables Using Continuous Wavelet Transform
}

\author{
M. Sreedevi and P. JenoPaul \\ Anna University, Tamil Nadu, India
}

\begin{abstract}
Medium and high voltage power cables are widely used in the electrical industry. This study describes about the implementation of continuous wavelet transform techniques to reject noise from on-line partial discharge measurements on cables. Different types of redundancy are detected and it is removed by the use of continuous wavelet transformation.
\end{abstract}

Key words: Partial discharge, continuous wavelet transform, redundancy, technique, medium, voltage power

\section{INTRODUCTION}

Partial discharges are small electrical sparks that occur within the electric insulation of switchgear, cables, transformers and windings in large motors and generators. Partial Discharge (PD) detection and classification is an important diagnostic tool for the reliable operation of power apparatus. In online partial discharge signal monitoring systems, one of the key problem is to identify PD's from the signals with strong disturbance and noise which are typical much stronger than PD's (Satish and Nazneen, 2003).

Traditionally, the main techniques used to reject noise from PD signals could be realized in either the time domain or in the frequency domain. However, when signal processing is done in the frequency domain, the time domain information is lost. The Wavelet Transform (WT) technique provides a new analysis tool able to be implement both in time and frequency domain.

\section{TYPES OF PARTIAL DISCHARGE}

Corona discharge: Sharp points inside the construction of the transformer especially on the high voltage side can produce discharges which are known as corona.

Surface discharge: This happens when there is a high field component parallel to the dielectric surface. As a result of these discharges, the dielectric material deteriorates and this can lead to erosion of the dielectric surface which eventually leads to breakdown.

Floating discharge: Badly earthed or floating parts can be present in a transformer. These parts do not have a defined potential and this can result in discharges.
A laboratory study is done by making the models of these discharges. Both time and frequency domain measurements are to be done (Ming and Birlasekaran, 2002).

\section{PD MEASUREMENT}

A variable $\mathrm{HV}$ source up to $7.5 \mathrm{kV}$ is used to energize the object with a protecting resistor. The HV is applied on the fabricated test objects and emitted PD signals are measured across $50 \Omega$ resistor using a high voltage coupling capacitor is shown in Fig. 1. A high frequency buffer differential amplifier is used to eliminate the dominant $50 \mathrm{~Hz}$ signal. The obtained signal is recorded on a 2 channel digital Agilent oscilloscope (6032A) at a sampling rate of 2 Giga samples $\mathrm{sec}^{-1}$. Then, it is interfaced with PC using GPIB interface. Necessary software routines are to be developed to control the GPIB controlled instruments and to acquire the data with proper settings. The collected data is analyzed using developed Matlab software routines (Cui and Huang, 2008).

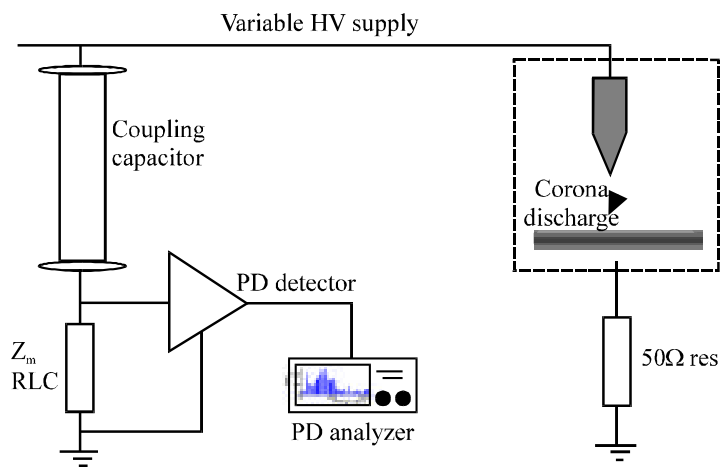

Fig. 1: Experimental setup 


\section{WAVELET DE-NOISING TECHNIQUE}

Decomposition: The general wavelet de-noising procedure involves three steps (Zhang et al., 2007). Choose a wavelet, choose a level $\mathrm{N}$ and compute the wavelet decomposition coefficients of the signals at levels from 1-N.

Select threshold detail coefficients: For each level from $1-\mathrm{N}$, select a threshold and apply a soft or hard threshold to the detail coefficients.

Reconstruction: To reconstruct signal by using original approximation coefficients and modified detail coefficients from levels 1-N.

\section{CONTINUOUS WAVELET TRANSFORM (CWT)}

The continuous wavelet transforms which transforms a continuous function in to a highly redundant function of two continuous variables. The resulting transform is easy to interpret and valuable for time-frequency analysis. The continuous wavelet transform of a continuous square integrable function $\mathrm{f}(\mathrm{x})$ relative to a real valued wavelet $\Psi$ (x) (Boggs and Densley, 2000). The continuous transform is similar to a series expansion or discrete transform. It provides both spatial and frequency information. The transform provides an objective measure of the similarity between $\mathrm{f}(\mathrm{x})$ and the wavelets for which it is computed.

\section{PROPOSED TECHNIQUE}

The conventional wavelet denoising method involves calculation of the CWT coefficients for a given signal and then passing the CWT through a threshold and followed by reconstruction of the signal by taking the inverse wavelet transform of the modified CWT coefficients. This method is known as soft or hard threshold.

Inter pixel redundancy: The gray levels in the images are not equally probable. Variable length coding can be used to reduce the coding redundancy that results from a straight or natural binary encoding of their pixels. The codes used to represent the gray levels of each image have nothing to do with the correlation between pixels. These correlation result from the structural or geometric relationships between the objects in the image.

Psychovisual redundancy: The eye does not respond with equal sensitivity to all visual information. The information simply has less relative importance than other information in normal visual processing. This information is said to be psychovisual redundancy. It can be eliminated without in pairing the quality of image perception.
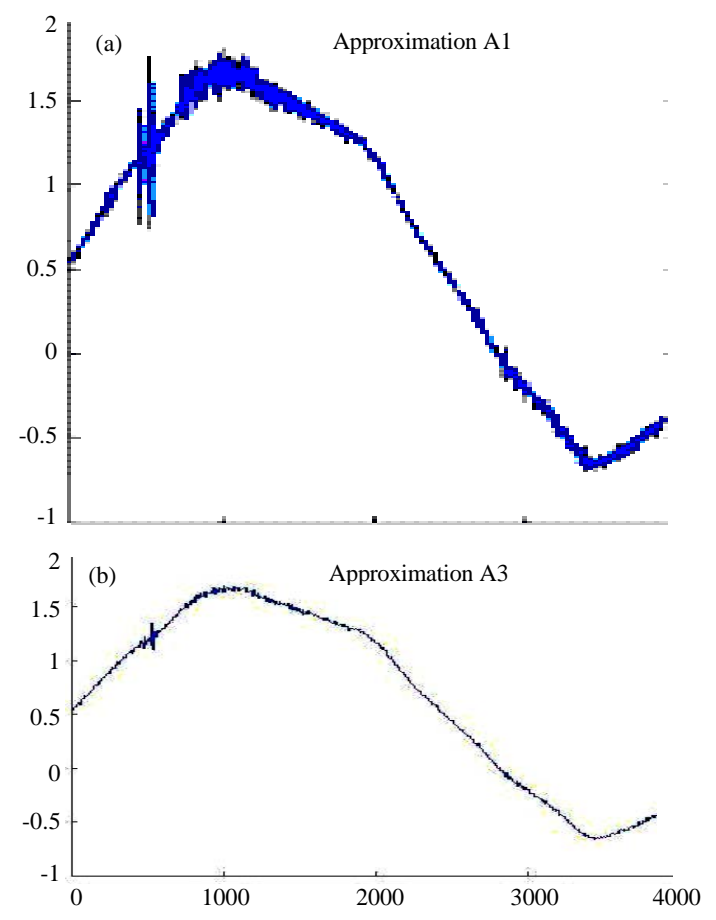

Fig. 2: (a) Approximation A1 outputs; (b) Approximation A3 outputs

Fidelity: When the level of information loss can be expressed as a function of the input image and the compressed and the decompressed output image is said to be fidelity criteria. The example is the root mean square error between input and the output image.

The proposed technique is basically an off-line technique and has the advantages of low processing time and possibility of better reconstruction because the suppression of noise/interferences is done in a joint time frequency domain. This type of denoising consists of two phases.

In the first phase, input signal is decomposed into approximate and detail components up to a desired number of levels with the help of multiresolution analysis approximate and detail components up to a desired number of levels with the help of multiresolution analysis. This is done by first choosing a mother wavelet according to the signal characteristics. Once, the mother wavelet is chosen, decomposition-reconstruction up to the required number of levels is carried out by scaling and dilating the mother wavelet.

Once the approximate and detail reconstructed time-domain components are computed, the second phase starts are shown in Fig. $2 \mathrm{a}$ and $\mathrm{b}$. This phase involves identifying those components that correspond to all the interference signals either by visual inspection or by 
the knowledge of frequency hands to which the PD pulses belong (this is known from the bandwidth of the $\mathrm{PD}$ detector). Finally, the denoised signal is obtained by discarding all the identified components from the summation process.

\section{NEED FOR SYNTHESIS OF INPUT SIGNAL}

For studying the performance of different noise suppression techniques, it is necessary to consider the influence of all the parameters of each method on the final result. These influences can critically be evaluated, if the input signals, noise/interferences, source and output signals are all exactly known.

In addition, the relevant parameters must easily be controllable. Hence, the input signal and interferences need to be synthesized in a realistic manner. This approach also facilitates the reproducibility of the results by other researchers.

Although, practical experimental setup is another source of such signals, the feasibility of controlling individual components and their relevant parameters is often time consuming and difficult, if not impossible.

\section{OUTPUT WAVEFORM}

The major problem that confronts on-line monitoring of PD activity in power cables is EM noise which can occur from a number of possible causes. While it is possible to detect high level PD signals in such situations using routine filtering techniques, the minimum PD magnitudes that are measurable with such techniques are so high that failure may be imminent.

To monitor cable condition and to be able to assess insulation degradation trends requires better sensitivity in $\mathrm{PD}$ detection and this requires better noise rejection techniques.

In the case of older XLPE cables where water trees are a problem, the transition from water trees to the detectable PDs of electrical trees is so rapid that alarm-based continuous on-line PD monitoring is needed to detect the onset of electrical trees and thus to prevent possible major cable failure is shown in Fig. 3.

Wavelet transform de-noising methods improve the capability to detect PDs signal in noisy environments. In this study a new method of wavelet processing has enabled using $30 \mathrm{pF}$. PD signals to be detected in noise levels higher than the PD signals. This has greatly increased the PD measurement sensitivity is shown in Fig. 4.

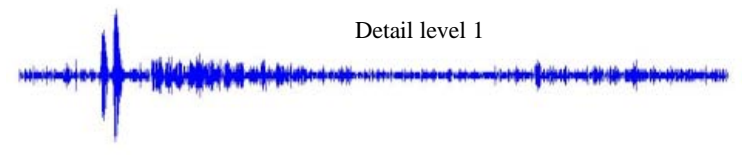

Detail level 2

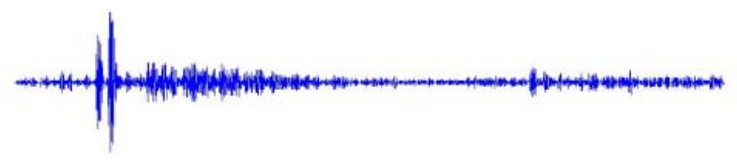

Detail level 3

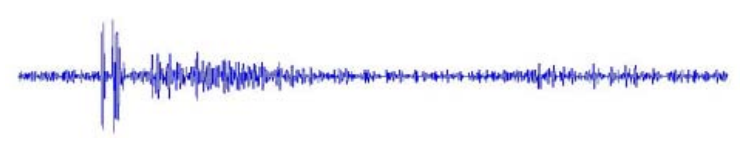

Fig. 3: Detailed outputs

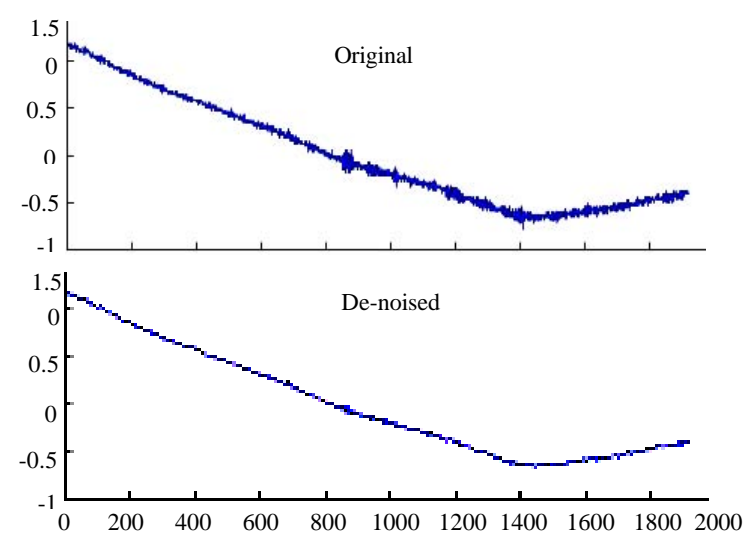

Fig. 4: Original and denoised PD signals

\section{REFERENCES}

Boggs, S. and J. Densley, 2000. Fundamentals of partial discharge in the context of field cable testing. IEEE Electr. Insul. Mag., 16: 13-18.

Cui, X. and T. Huang, 2008. A novel method of selecting complex wavelet for feature extraction in partial discharge signal processing. Proceedings of the Congress on Image and Signal Processing, May 27-30, Sanya, Hainan, pp: 128-131.

Ming, Y. and S. Birlasekaran, 2002. Characterization of partial discharge signals using wavelet and statistical techniques. Proceedings of the IEEE International Symposium on Electrical Insulation, April 7-10, Boston, MA. USA., pp: 9-13.

Satish, L. and B. Nazneen, 2003. Wavelet-based denoising of partial discharge signals buried in excessive noise and interference. IEEE Trans. Dielectr. Electr. Insul., 10: 354-367.

Zhang, H., T.R. Blackburn, B.T. Phung and D. Sen, 2007. A novel wavelet transform technique for on-line partial discharge measurements. 1. WT de-noising algorithm. EEE Trans. Dielectr. Electr. Insul., 14: 3-14. 